\title{
Fine-scale genetic structure and marginal processes in an expanding population of Biscutella laevigata L. (Brassicaceae)
}

\author{
C Parisod and G Bonvin \\ Department of Ecology and Evolution, Biophore-Sorges, University of Lausanne, Lausanne, Switzerland
}

\begin{abstract}
Evolutionary processes acting at the expanding margins of a species' range are still poorly understood. Genetic drift is considered prevalent in marginal populations, and the maintenance of genetic diversity during recolonization might seem puzzling. To investigate such processes, a fine-scale investigation of 219 individuals was performed within a population of Biscutella laevigata (Brassicaceae), located at the leading edge of its range. The survey used amplified fragment length polymorphisms (AFLPs). As commonly reported across the whole species distribution range, individual density and genetic diversity decreased along the local axis of recolonization of this expanding population, highlighting the enduring effect of the historical colonization on present-day diversity. The self-incompatibility system of the plant may have prevented local inbreeding in newly found
\end{abstract}

patches and sustained genetic diversity by ensuring gene flow from established populations. Within the more continuously populated region, spatial analysis of genetic structure revealed restricted gene flow among individuals. The distribution of genotypes formed a mosaic of relatively homogenous patches within the continuous population. This pattern could be explained by a history of expansion by longdistance dispersal followed by fine-scale diffusion (that is, a stratified dispersal combination). The secondary contact among expanding patches apparently led to admixture among differentiated genotypes where they met (that is, a reshuffling effect). This type of dynamics could explain the maintenance of genetic diversity during recolonization.

Heredity (2008) 101, 536-542; doi:10.1038/hdy.2008.95; published online 10 September 2008

Keywords: amplified fragment length polymorphism; high-altitude population; landscape genetics; recolonization; reshuffling effect; stratified dispersal

\section{Introduction}

Species range expansion is generally associated with decreasing allelic richness with increasing distance from the source populations; such patterns seem to have been generated by postglacial recolonization (Comps et al., 2001; Lascoux et al., 2004). In plants, the cumulative effects of the recurrent foundation of populations at the expanding species' margin by long-distance seed dispersal leads to the loss of genetic diversity along recolonization pathways at the continental scale and leaves a long-lasting genetic signature across species ranges (Hewitt, 1996, 2000). The maintenance of genetic diversity during expansion might, therefore, seem to be puzzling.

In contrast, Petit et al. (1997) showed that historical processes can result in a patchy genetic structure of recolonizing maternal lineages. Indeed, the independent foundation of nearby populations by long-distance seed dispersal followed by local diffusion (that is, stratified dispersal) can induce the clustered distribution of

Correspondence: Dr C Parisod, Institut J-P. Bourgin, Laboratoire de Biologie Cellulaire, INRA, Centre de Versailles, F-78026 Versailles, France.

E-mail: cparisod@versailles.inra.fr

Received 25 February 2008; revised 23 June 2008; accepted 27 July 2008; published online 10 September 2008 haplotypes and thereby increase regional diversity. Petit et al. (2003) also showed that the populations can exhibit increased heterozygosity away from refugia because of the admixture of previously allopatric lineages replenishing genetic diversity. In other words, long-distance seed dispersal not only affects the speed of recolonization, but may also influence the spatial genetic structure of expanding populations (LeCorre et al., 1997; Austerlitz et al., 2000). Recently, simulation studies have found that the intermediate rates of long-distance seed dispersal generate patchiness and, throughout breeding, maintain genetic diversity against the depressing effect of recurrent founder events (Bialozyt et al., 2006).

Although the population dynamics at the margins of a species' range is crucial to understanding the limits of its distribution and its response to environmental changes, empirical population studies usually consider edges as a nuisance (Kirkpatrick and Barton, 1997). Most studies focus on the central-marginal distinction at the regional scale and ambiguously quantify the historical versus the contemporary factors affecting the structure of genetic variation. Accordingly, more than half of the studies that test for genetic diversity in both plants and animals report the expected decline of a within-population genetic diversity towards range margins (Eckert et al., 2008). However, the putative evolutionary processes affecting marginal populations remain largely theoretical. In fact, the dynamics of expanding 
populations is most probably dominated by environmental gradients and gene dispersal (Lönn and Prentice, 2002). Dispersal is important, because it governs the amount of seeds landing into suitable habitat, but it also shapes genetic diversity in leading edge populations through gene flow mediated by seeds and pollen (Austerlitz and Garnier-Géré, 2003). Ultimately, gene flow determines the possible evolution of ecological tolerance in marginal areas (Antonovics et al., 2001; Lenormand, 2002).

Alpine landscapes provide great opportunities to understand the genetic consequences of recolonization and the evolutionary processes acting on marginal populations, because elevation causes sharp ecological gradients on the local scale (Herrera and Bazaga, 2008). Thus, sampling across the edge of the species' range is possible on a local scale, and dynamic processes can be inferred. This study investigates a local, nearly continuous population of Biscutella laevigata (Brassicaceae) along an elevation gradient up to the species altitudinal margin. It builds on our knowledge of the historical context provided by a preceding phylogeographical study of the region (Parisod and Besnard, 2007). It surveys the ecology and the genetic diversity of 219 individuals with amplified fragment length polymorphisms (AFLPs), and aims to (i) test the importance of historical recolonization and (ii) highlight its consequences on the fine-scale distribution of individuals as well as their genetic diversity. Recolonization has left a slight cline of genetic diversity at the local scale, but also resulted in a patchy genetic structure, suggesting that the genetic diversity is maintained despite the founder effect through stratified dispersal and admixture.

\section{Materials and methods}

\section{The study species}

Biscutella laevigata L. (Brassicaceae) has expanded outside the Mediterranean basin to become a true (ad-)alpine taxon (Schönfelder, 1968). It is a long-lived perennial and outcrossing species with a sporophytic self-incompatibility system (Olowokudejo and Heywood, 1984). Pollen dispersal is achieved by generalist Diptera and Lepidoptera, and seeds are mainly dispersed by gravity and wind. After the early studies of Manton (1937), B. laevigata was considered to have survived the Pleistocene vicissitudes as a diploid $(2 n=2 x=18)$ in ice-free parts of the European continent and to have recolonized the Alps as an autotetraploid $(2 n=4 x=36)$. More recently, plastid DNA phylogeography has suggested a glacial survival in multiple refugia within the Alps and has indicated that the species evolved through polytopic autopolyploidy; therefore, that independent lineages recolonized the Alps (Parisod and Besnard, 2007). One of the autotetraploid lineages (E) has expanded widely over the previously glaciated areas of the northern Alps and exhibits reduced genetic diversity in the central Alps (Parisod, 2008). Well within the area covered by this lineage, a derivedhaplotype with a characteristic chloroplastic microsatellite variation of postglacial origin (Ea; Parisod and Besnard, 2007), has undergone a restricted extension in the Massif des Diablerets (that is, over the present study area).
Sampling site and strategy

The sampling site is a hanging valley between 2000 and $2525 \mathrm{~m}$, located in the Massif des Diablerets of the western Swiss Alps, close to permanent glaciers $\left(7^{\circ} 12^{\prime}\right.$; $46^{\circ} 20^{\prime}=$ Swiss coordinates: 583'000; 132'300). This U-shaped valley is considered highly isolated from the surrounding landscape because of the cliffs that run alongside it, which are greater than $200 \mathrm{~m}$ in height (Figure 1). The population of B. laevigata is dense and nearly continuous between 2000 and $2300 \mathrm{~m}$ and, in the upper part of the valley, the species occurs in small patches and as isolated individuals until the top of the valley $(2525 \mathrm{~m})$. Ecological surveys (species composition, soil description and habitat modelling; $C$ Parisod and S Joost, unpublished data) indicate that the valley is characterized by a mosaic of typical high-altitude habitats on calcareous substrates, and show that $B$. laevigata lies exclusively in the warmer and drier meadows of the valley. The species avoids wet or cold areas (for example, pergelisol), but otherwise grows in diverse habitats that are mainly differentiated by total radiation.

The valley was physically divided into $100 \times 100 \mathrm{~m}$ squares that were further subdivided by a $25 \times 25 \mathrm{~m}$ grid whose intersections were checked for the presence of $B$. laevigata. The species was censused in $2 \times 2 \mathrm{~m}$ quadrats and, when 10 individuals were present, the leaves of the four individuals located nearest to each corner were collected and dried in silica gel to give a $25-\mathrm{m}$ grid sample (hereafter called plot). The location of each plot was determined in the field using the physical grid and

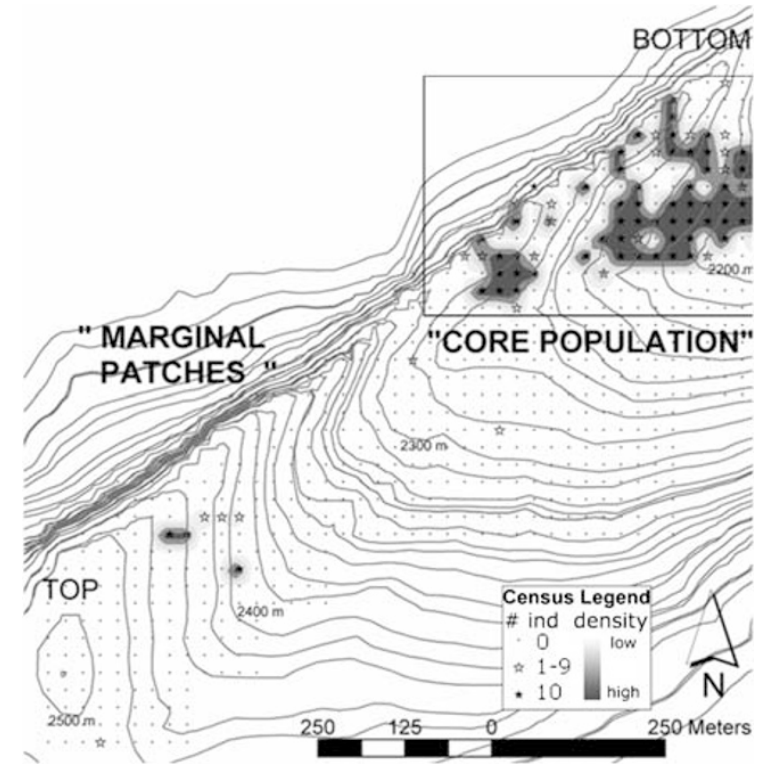

Figure 1 Distribution of the population of Biscutella laevigata over the sampling area. The base map presents iso-elevation lines. Individual census in $2 \times 2 \mathrm{~m}$ has been recorded every $25 \mathrm{~m}$ (absence represented by black dots and presence by stars; black stars show plots with 10 individuals that have been used for genetic analyses and plots with less than 10 individuals are shown by empty stars). The number of individuals within plots has been spatially interpolated over the area, the darker the zone the denser the population. The population is divided into a dense 'core population' of two parapatric patches and allopatric 'marginal patches' at the top of the valley. The area in the rectangle represents the portion of the population that is presented in Figure 3. 
confirmed by a Garmin GPS unit with a precision of less than $10 \mathrm{~m}$. The species was present in 69 sampling points out of 720 , which were used to analyse the distribution. To give a representation of the density throughout the study area, spatial interpolation was used (inverse distance weighted interpolation of power 2), using the nearest neighbour resampling method with a radius including 12 plots as implemented in Environmental Systems Research Institute, ArcGIS 8.1. Only 54 points showed more than 10 individuals in $2 \times 2 \mathrm{~m}$ and these 216 individuals as well as three isolated individuals at the top of the valley were sampled to give a dataset of 219 individuals for the genetic analyses.

Fruit numbers and rosette size (length $\times$ width) of each sampled individual were measured in the field and an index of reproductive investment was calculated using the ratio of fruit number to rosette surface area. Additionally, environmental values were extracted for the location of every plot from the geographic information system eco-climatic layers of Zimmermann and Kienast (1999). These were used as explanatory variables to explore their contribution to the average reproductive investment per plot using stepwise multiple regressions with randomly ordered variables. On the basis of the species habitat model (S Joost and C Parisod, unpublished data), the following ecological factors were used. They were chosen because they varied within the habitat of $B$. laevigata in this study (the range is shown in brackets) and were shown to influence the genetic architecture of populations (Parisod and Christin, 2008). The variables were: degree-days during the growing season (735-921 days degree), elevation (2204-2433 m), number of frost days during growing season (8.9-18.1 days with frost), slope $\left(7-56^{\circ} \mathrm{C}\right)$ and total solar radiation (17763-63928 kJ/day).

\section{Genetic analysis}

Total DNA was extracted from dried leaf tissue using the FastDNA kit (Q-Biogen, Carlsbad, CA, USA) in a room devoted to DNA extraction. The manufacturer's instructions were followed except that extracted DNA was washed twice. DNA quality and concentration were checked on agarose gels.

Amplified fragment length polymorphism markers were generated following the AFLP Plant Mapping Kit protocol (Perkin-Elmer Applied Biosystems, Foster City, CA, USA) using $150 \mathrm{ng}$ of genomic DNA. This protocol provided ready to use solutions to standardize concentration fluctuations and maximize reproducibility. Restrictions, ligations and amplifications were always performed on the same Biometra-T3 thermocycler. Eight selective primer combinations were tested independently three times on 10 individuals to check for polymorphisms and reproducibility. The whole AFLP procedure was replicated twice on 10 individuals and an error rate was calculated as the number of differences among profiles of the same individual divided by the total number of fragments scored (Bonin et al., 2004). On the basis of this preliminary survey, two combinations (MCAG/E-ACA and M-CTC/E-AGG, with the fluorescent E primer labelled by FAM and JOE, respectively) were selected to generate AFLP markers on 219 individuals. PCR products were visualized using an ABI-PRISM 377 sequencer on $6 \%$ long ranger denaturing gel for $5 \mathrm{~h}$
(2000 V, $50 \mathrm{~A})$. Fragment sizes were estimated using the 500-ROX standard and were scored manually as dominant markers, using the Genescan 3.1.2 software (Applied Biosystems). Only clear bands with high peaks were scored, whereas weak profiles were discarded.

\section{Statistical analysis}

Most of the population genetic analyses were performed on the presence/absence matrix of AFLP fragments from the continuous part of the population (the 'core population' with 204 individuals, Figure 1). Individuals from locations with higher elevation ('marginal patches' with 15 individuals) were compared with those of the continuous population.

At the individual level, the spatial genetic structure in two dimensional space was explored by spatial autocorrelation methods (Vekemans and Hardy, 2004). The relationship between the pairwise relationship coefficients on the basis of AFLP profiles and the logarithm of geographic distance among individuals was quantified using SPAGeDi (Hardy and Vekemans, 2002; Hardy, 2003). An autocorrelogram was constructed to explore the genetic relationship among genotypes at increasing geographical intervals with a balanced number of individuals.

At the plot level, the Shannon diversity index based on AFLP profiles in each 25-m plot was computed according to Parisod and Christin (2008), to analyse patterns of genetic diversity in the population. The relationship between the Shannon diversity index and randomly ordered environmental values was explored by stepwise multiple regression. The association between the distance from the bottom of the valley and the Shannon genetic diversity was examined by robust regression, using S-plus. Given that Hardy-Weinberg equilibrium was not expected in this autotetraploid population of $B$. leavigata, a between-group Eigenanalysis (BPCA, that is, principal component analysis among plots based on principal component analysis on the covariance matrix among AFLP profiles) was performed according to Parisod and Christin (2008). This band-based Euclidian approach summarized and partitioned a maximum of genetic variance into few dimensions, giving $\beta_{\mathrm{ST}}$ estimates of the genetic structure among plots. Although possibly inflated, relative $\beta_{\mathrm{ST}}$ values have been shown to be related to traditional F-statistics (Parisod et al., 2005; Parisod and Christin, 2008). The BPCA analysis was performed among 25-m plots as well as individuals combined into the $100-\mathrm{m}$ squares used for field sampling. Significance was estimated by a 9999 permutation test using ADE-4 (Thioulouse et al., 1998). In addition, BPCA produced univariate genotypic variables (BPCA scores) that could be analysed further with spatial statistics. To give a representation of the genetic composition throughout the core population, BPCA plot scores were spatially interpolated (inverse distance weighted interpolation of power 2), using the nearest neighbour resampling method with a radius including 12 plots as implemented in Environmental Systems Research Institute, ArcGIS 8.1. The relationship between the Shannon diversity of plots and the first BPCA score was explored by a generalized additive model (Hastie and Tibishirani, 1986). These models assume that the mean of the dependent variable depends on an additive predictor through a nonlinear 
link function. Generalized additive models are designed to avoid postulating a specific parametric response function and use smoother functions that attempted to generalize data into curves by local fitting. Using S-plus, the value of the dependent variables along a single variable was plotted and a parsimonious smooth curve fitting the data as closely as possible was calculated with epsilon $=0.001$.

\section{Results}

\section{Distribution}

The spatial interpolation of the individual census at each plot showed that the continuous population of $B$. laevigata was not homogeneously distributed across the valley. A 'core population' showing dense patches at the bottom of the sampling area, with 41 plots containing 10 individuals (density, 2.5 individuals $/ \mathrm{m}^{2}$ ) as well as a parapatric area with 10 plots of similar density located at slightly higher elevation can be distinguished (Figure 1). In addition, 'marginal patches' comprising two and one isolated plots respectively, as well as three isolated individuals, were located at the top of the valley. Although the reproductive investment among plots was significantly different (Kruskal-Wallis $=88.802$, $P=0.003)$, none of the environmental values tested by stepwise multiple regression significantly explained the plot mean investment $\left(P=0.129 ; r^{2}=0.044\right.$; Supplementary Table S1).

\section{Genetic analysis}

On the basis of the replication of the AFLP protocol on 10 individuals, the error rate was estimated to be $2.4 \%$, and 113 polymorphic fragments were reliably scored within the population.

Fine-scale genetic structure was detectable among individuals as the autocorrelogram of spatial pairwise relationship coefficients based on inter-individual AFLP profiles showed that geographically nearby individuals are genetically related (Figure 2). Only the distance class 1 (mean, 31.9 and maximum, 54.1) and class 2 (mean, 67.7 and maximum, 80.1) had a significant relationship

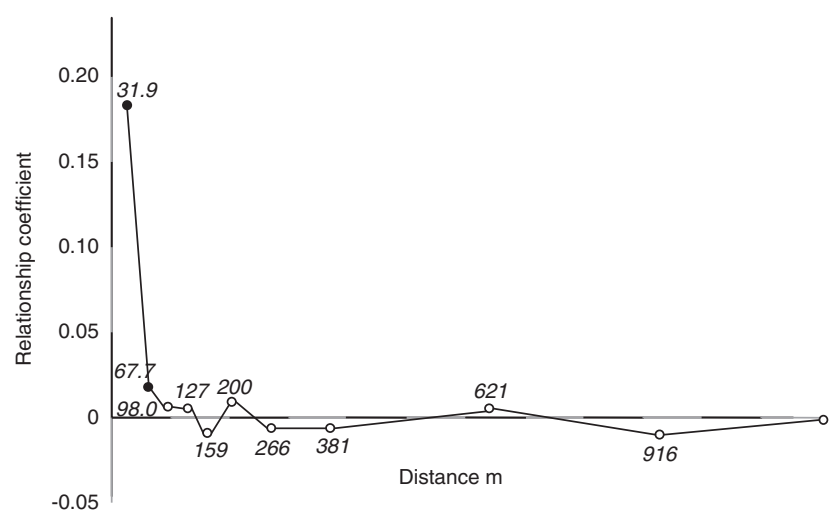

Figure 2 Fine-scale spatial genetic structure in the high-altitude continuous population of Biscutella laevigata. Autocorrelogram presenting the mean relationship coefficient in 10 balanced logdistance classes, whose average values are presented in italics. The distance scale is linear. Black dots represent mean values of pairwise relationship coefficients for distance classes that are significantly different from zero; empty dots represent nonsignificant values. coefficient (0.184 and 0.011 , respectively), whereas larger distance classes showed no significant values.

At the plot level, the average within-plot Shannon index of genetic diversity was 0.270 ( \pm 0.057 ; Supplementary Table S1). Genetic diversity was not significantly explained by any environmental factor tested in stepwise multiple regression $\left(P=0.278, r^{2}=0.048\right)$. Neither Shannon diversity, nor reproductive investment was significantly different between the core population plots and those of the marginal patches (Wilcoxon rank sum test: $P=0.184$ and $P=0.430$, respectively). However, the genetic diversity within the core population significantly decreased with increasing geographic distance from the bottom of the valley (Shannon index $=-0.0002$ distance $\left.=+0.4350, r^{2}=0.2024, P=0.0016\right)$. As indicated by BPCA, the genetic structure among the 25-m plots was significant $\left(\beta_{\mathrm{ST}}=0.32, \quad P<0.001\right)$, showing that most of the genetic variance was partitioned between sampling plots. The same analysis among plots combined into the 100-m squares showed a reduced amount of the genetic structure at a broader spatial scale $\left(\beta_{\mathrm{ST}}=0.08, P<0.001\right)$, showing that most of the genetic variance was within $100-\mathrm{m}$ squares. The spatial extrapolation of the first BPCA score showed a complex spatial architecture of genetic variation within the core population, forming a mosaic of different genotypes patchily distributed over the valley. Indeed, genetically similar plots were shown to be spatially clustered with sharp transitions to neighbouring plots, forming genetically homogeneous patches made up of different genotypes (Figure 3). In addition, several high-diversity plots were located at the contact zone among genetically

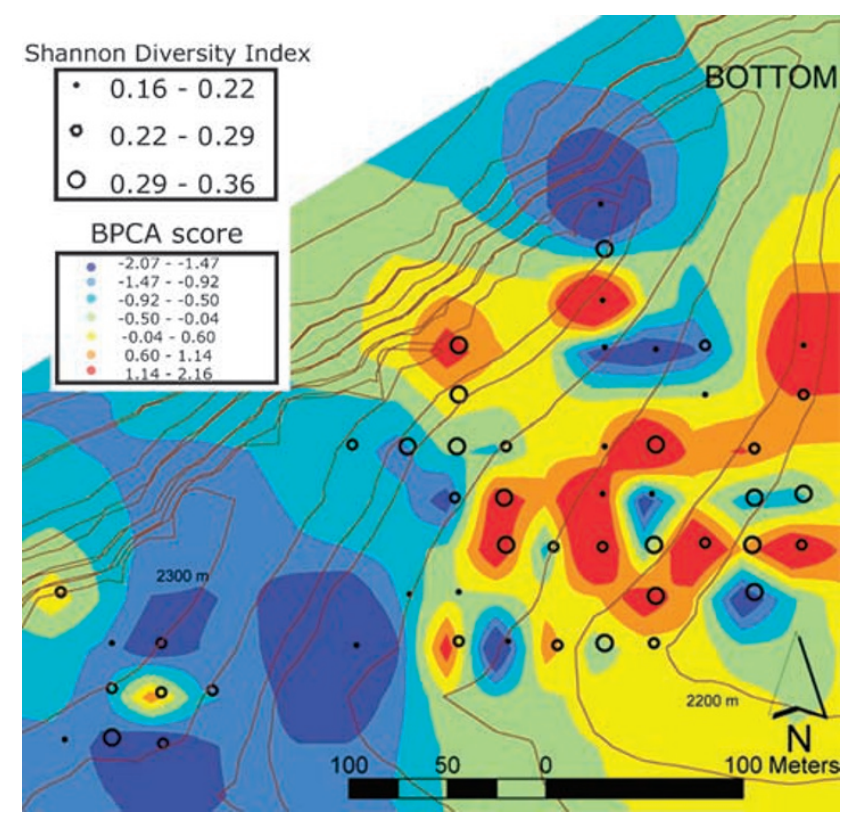

Figure 3 Genetic architecture of the high-altitude population of Biscutella laevigata. The spatial interpolation of the first betweengroup Eigenanalysis (BPCA) score among plots over the core population is shown. Colours correspond to classes of quantiledBPCA scores detailed in the panel. Plots are marked with circles, which represent the within-plot Shannon index of genetic diversity in three equal classes (according to the panel: small, medium and big circles represent low, intermediate and high genetic diversity, respectively). Note that most of the high-diversity plots are located at the contact zone among genetically homogeneous patches. 
540

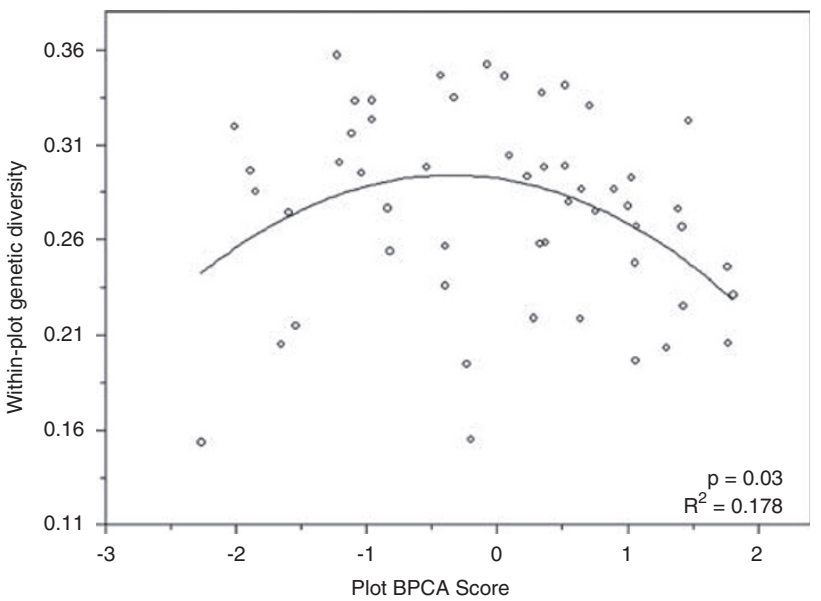

Figure 4 Relationship between the within-plot Shannon index of genetic diversity and the first between-group Eigenanalysis (BPCA) score of amplified fragment length polymorphism (AFLP) profiles among plots. The Gaussian additive model is significant $(P=0.03$, deviance explained $=0.178$ ) and indicates that plots with intermediate AFLP profiles show a significantly higher genetic diversity than the extreme ones forming homogeneous patches. Such a pattern is expected for nuclear genes under a 'reshuffling effect'.

dissimilar patches. The generalized additive model between the Shannon diversity within plots and their first BPCA score indicated a significant relationship (Figure 4; $P=0.03$; deviance explained $=0.178$ ). The genetic diversity was thus shown to be consistently greatest when BPCA scores were intermediate that spatially corresponded to transitions among homogeneous patches (Figure 3).

\section{Discussion}

The phylogeography of $B$. laevigata shows that postglacial recolonization results in a reduced haplotype diversity within maternal lineages of the internal Alps compared with the external Alps (Parisod and Besnard, 2007). Local populations investigated here in the internal Alps are fixed with a characteristic plastid haplotype of postglacial origin, which further suggests that the founder effect was strong during recolonization. The populations studied here are in the vicinity of permanent glaciers, at the altitudinal margin of vegetation, and certainly correspond to the local recolonization front (Ackerley, 2003; Raffl et al., 2006). As the dynamics of leading edge populations has not been extensively investigated on the local scale (Coart et al., 2005; Eckert et al., 2008), the distribution and genetic patterns supporting putative expansion have to be documented before addressing the evolutionary processes acting on recolonizing populations.

\section{Polarised recolonization of the valley}

The distribution pattern observed here may be considered typical of the leading edge of an expansion (Thomas et al., 2001). The census analysis shows that the population of $B$. laevigata can be divided into a core population of two parapatric patches of unequal extent, plus an area at higher elevation presenting small patches and isolated individuals. These marginal patches at the top of this highly isolated valley are widely allopatric (more than $600 \mathrm{~m}$ apart of the core population; Figure 1), suggesting that they have been founded by successful seed dispersal from patches lying at lower elevation. Furthermore, B. laevigata is locally abundant in the warmer areas of the valley, but local habitats are apparently unsaturated as favourable zones around the altitude margin are unoccupied (Figure 1). There was no significant evidence that ecological factors explain the differences of reproductive investment among plots, suggesting that the demography is not constrained by environmentally induced variation in fitness. Accordingly, the survey indicates that either local adaptation or historical constraints are responsible for the distribution of individuals (Kluth and Bruelheide, 2005).

Genetic evidence supports the historical scenario and indicates that recolonization has strongly influenced this marginal population. Actually, the Shannon index of genetic diversity shows a slight but significant decrease in genetic diversity from the bottom to the top of the core population. Declining genetic diversity with increasing distance from source population is a common phylogeographic pattern on the regional scale, reflecting the recurrent founder effect affecting expanding populations (Hewitt, 2000; Comps et al., 2001). The cline of genetic diversity observed here on the local scale thus corroborates the polarised recolonization from the bottom towards the top of the valley that can be inferred from the distribution data. To our knowledge, such a pattern on the local scale has never been reported before. The signatures of local nonequilibrium process have probably detected because of the following properties of the study system. Biscutella laevigata is a long-lived perennial with thermophilous ecological requirements, and the study area has been recently glaciated. The limited time span as its foundation may explain why historical patterns are still detectable (Austerlitz et al., 2000); the present population can be confidently assumed to be younger than thousand generations. Furthermore, the landscape features of the study site, including the steep elevation gradient over short geographical distances and the high bordering cliffs impeding recurrent immigration, have allowed for a nonambiguous interpretation of the observed patterns as a consequence of recolonization. Historical processes can therefore be regarded as having a long-lasting impact on local populations, this fine scale-and should be considered when interpreting the genetic patterns.

\section{Genetic structure: marginal processes and stratified recolonization}

As the dense sampling adopted here, details about the evolutionary dynamics of this expanding population can be inferred. Inter-individual relationship coefficients indicate that the continuous population is characterized by an important fine-scale genetic structure (Vekemans and Hardy, 2004). Indeed, only individuals in the first two distance classes (below $81.1 \mathrm{~m}$ ) exhibit a genetic relationship significantly higher than zero (Figure 2), indicating that gene dispersal is restricted. Congruently, F-statistics among 25-m plots demonstrate genetic structure within the continuous population $\left(\beta_{\mathrm{ST}}=0.32\right)$. As isolation by distance is lacking among plots, the observed genetic structure matches nonequilibrium 
expectations and suggests that the continuous population has undergone dynamic expansion (Slatkin, 1993; Hewitt, 2000).

The marginal patches have been founded recently, providing the opportunity to understand the early evolutionary processes acting on leading edge populations. The founder effect is expected to lead to a drastic reduction in the genetic diversity within allopatric patches and to an increased genetic differentiation with the core population (Hewitt, 1996). Here, the marginal patches are genetically well differentiated from one another, suggesting independent founder events, but not significantly differentiated from the core population, suggesting gene flow. In contrast to the observed cline of genetic diversity within the core population, the marginal patches have a similar genetic diversity to the core population $(P=0.184)$. Similarly, other comparable studies on the local scale (Yang et al., 2008) or in mountainous landscape (Raffl et al., 2006; Herrera and Bazaga, 2008) have reported that genetic diversity is not lower, and can even be higher, in marginal populations. The mating system and the perennial life history of $B$. laevigata may have helped to maintain genetic diversity despite founder effects. The species has a sporophytic self-incompatibility system (Olowokudejo and Heywood, 1984), implying that isolated individuals cannot self or reproduce with close relatives and are confronted with limited mate availability (C Parisod, personal observation). Marginal colonizers are therefore dependant on long-distance pollen dispersal from neighbouring patches to find compatible mates after foundation. Immigration of unrelated genotypes is thus crucial until allopatric patches achieve sufficient genetic variation at the incompatibility locus (Goodell et al., 1997). The high genetic diversity observed in the marginal patches suggests that the early colonizers of $B$. laevigata transiently act as a sink of genetic diversity through pollen flow, thus replenishing the genetic diversity of marginal patches during recolonization (Austerlitz and Garnier-Géré, 2003).

Compared with the fine-scale level in the core population $\left(\beta_{\mathrm{ST}}\right.$ among plots $\left.=0.32\right)$, the genetic structure when individuals are aggregated into $100-\mathrm{m}$ squares is low $\left(\beta_{\mathrm{ST}}=0.08\right)$. This discrepancy among genetic structure at different spatial scales indicates that the valley has not been recolonized by a diffusion-like process, which would have caused genetic homogeneity at both scales (Ibrahim et al., 1996; Bialozyt et al., 2006). Instead, the observed pattern suggests stratified dispersal, which means that the valley has been recolonized by rare seed dispersal away from the mother plant, followed by local diffusion (Davies et al., 2004). Under restricted diffusion, infrequent seed dispersal outside the neighbourhood would have promoted the foundation of nearby patches by distantly related individuals as well as distant patches by related individuals. Congruently, the spatial distribution of genotypes shows that the core population of $B$. laevigata is a mosaic of differentiated patches displaying genetically related individuals (Figure 3). The spatial extent of homogeneous patches is strongly sensitive to the distance covered by dispersing propagules (Ibrahim et al., 1996) as well as the rate of long-distance dispersal events (Davies et al., 2004). Here, genetically homogeneous patches are relatively small in size, suggesting that the dispersal away from relatives occurs at an intermediate rate and that none of the founded patches have had the time to grow extensively before they merge into a continuous population.

By repeatedly founding new patches, stratified recolonization can have important consequences on genetic diversity. Indeed, expansion can promote the admixture among independently founded, differentiated gene pools, thereby elevating genetic diversity (Davies et al., 2004; Bialozyt et al., 2006). This phenomenon, referred to as the 'reshuffling effect', has been supported by simulation studies and may participate in the maintenance of maternally inherited plastid genetic diversity (Bialozyt et al., 2006). Accordingly, individuals lying in contact zones among homogeneous patches are expected to show a higher probability of having recombinant genotypes at nuclear markers than those lying within patches. In the study population, a significant Gaussian relationship between the genetic diversity and the first BPCA score shows that plots with intermediate AFLP profiles (that is, those lying in the centre of the BPCA reduced space) also have a higher genetic diversity (Figure 4). This pattern suggests that the intermediate genotypes have been produced by admixture of genetically differentiated individuals (that is, those with extreme BPCA scores), which increases heterozygosity and genetic diversity measured by AFLPs (Petit et al., 2003). These high-diversity plots are consistently situated at the contact zone among homogeneous patches (Figure 3), suggesting that the admixture among independently found patches shapes the diversity of nuclear markers according to the 'reshuffling effect'. Indeed, the fine-scale pattern observed within this expanding population of $B$. laevigata points to a reorganization of the architecture of genetic variation following stratified dispersal, which could have supported the maintenance of nuclear gene diversity despite recurrent founder effects.

In contrast to another population of B. laevigata, where solar radiation seems to explain the distribution of genetically homogeneous patches (Parisod and Christin, 2008), no association between the genetic structure and the environmental heterogeneity has been revealed here (C Parisod, unpublished data). This suggests that the genotypes are randomly distributed according to the abiotic environment in this expanding population. The maintenance of genetic diversity despite founder effects during recolonization therefore seems to be jointly explained by the species mating system, which encourages long-distance pollen flow, and the intermediate rate of long-distance seed dispersal, which promotes admixture among genetically divergent individuals. This fine-scale investigation thus sheds light on dynamic processes related to range expansion, showing that leading edge populations may not be passive sites of genetic depauperation, but could entertain evolutionary processes that sustain genetic diversity.

\section{Acknowledgements}

We thank E McCarthy for English improvements, P-A Christin, N Galland, R Holderegger, S Joost and N Maunoury, as well as anonymous reviewers for their helpful comments on the manuscript. We thank F Bancala and C Trippi for their help in the field, O Broennimann for statistical advices on GAMs and the Société Académique vaudoise for funding. 


\section{References}

Ackerley D (2003). Community assembly, niche conservatism, and adaptive evolution in changing environments. Int J Pl Sci 164: S165-S184.

Antonovics J, Newman TJ, Best BJ (2001). Spatially explicit studies on the ecology and genetics of population margins. In: Silvertown J, Antonovics J (eds). Integrating Ecology and Evolution in a Spatial Context. Blackwell Science: Oxford. pp 97-116.

Austerlitz F, Garnier-Géré P (2003). Modelling the impact of colonisation on genetic diversity and differentiation of forest trees: interaction of life cycle, pollen flow and seed long-distance dispersal. Heredity 90: 282-290.

Austerlitz F, Mariette S, Machon N, Gouyon P-H, Godelle B (2000). Effects of colonisation processes on genetic diversity: differences between annual plants and tree species. Genetics 154: 1309-1321.

Bialozyt R, Ziegenhagen B, Petit RJ (2006). Contrasting effects of long distance seed dispersal on genetic diversity during range expansion. J Evol Biol 19: 12-20.

Bonin A, Bellmain E, Bronken-Eidesen P, Pompanon F, Brochmann C, Taberlet P (2004). How to track and assess genotyping errors in population genetics studies. Mol Ecol 13: 3261-3273.

Coart E, Van Glabeke S, Petit RJ, Van Bockstaele E, Roldan-Ruiz I (2005). Range wide versus local patterns of genetic diversity in hornbeam Carpinus betulus L. Conserv Genet 6 259-273.

Comps B, Gömöry D, Letouzey J, Thiébaut B, Petit RJ (2001). Diverging trends between heterozygosity and allelic richness during postglacial colonization in the European beech. Genetics 157: 389-397.

Davies S, White A, Lowe A (2004). An investigation into effects of long-distance seed dispersal on organelle population genetic structure and colonisation rate: a model analysis. Heredity 93: 566-576.

Eckert CG, Samis KE, Lougheed SC (2008). Genetic variation across species' geographical ranges: the central-marginal hypothesis and beyond. Mol Ecol 17: 1170-1188.

Goodell K, Elam DR, Nason J, Ellstrand NC (1997). Gene flow among small populations of a self-incompatible plant: an interaction between demography and genetics. Am J Bot 84: 1362-1371.

Hardy OJ (2003). Estimation of pairwise relatedness between individuals and characterization of isolation-by-distance processes using dominant genetic markers. Mol Ecol 12 $1577-1588$.

Hardy OJ, Vekemans X (2002). SPAGeDi: a versatile computer program to analyse spatial genetic structure at the individual or population levels. Mol Ecol Notes 2: 618-620.

Hastie T, Tibishirani R (1986). Generalized additive models. Stat Sci 1: 297-318.

Herrera CM, Bazaga P (2008). Adding a third dimension to the edge of a species' range: altitude and genetic structuring in mountainous landscapes. Heredity 100: 275-285.

Hewitt GM (1996). Some genetic consequences of ice ages, and their role in divergence and speciation. Biol J Linn Soc 58: 247-276.

Hewitt GM (2000). The genetic legacy of the quaternary ice ages. Nature 405: 907-913.

Ibrahim KM, Nichols RA, Hewitt GM (1996). Spatial patterns of genetic variation generated by different forms of dispersal during range expansion. Heredity 77: 282-291.
Kirkpatrick M, Barton NH (1997). Evolution of a species' range. Am Nat 150: 1-23.

Kluth C, Bruelheide H (2005). Central and peripheral Hornungia petraea populations: patterns and dynamics. J Ecol 93: 584-595.

Lascoux M, Palmé AE, Cheddadi R, Latta RG (2004). Impact of ice ages on the genetic structure of trees and shrubs. Phil Trans Roy Soc Lond Series B 359: 197-207.

LeCorre V, Machon N, Petit RJ, Kremer A (1997). Colonization with long-distance seed dispersal and genetic structure of maternally inherited genes in forest trees: a simulation study. Genet Res 69: 117-125.

Lenormand $T$ (2002). Gene flow and the limits to natural selection. Trends Ecol Evol 17: 183-189.

Lönn M, Prentice HC (2002). Gene diversity and demographic turnover in central and peripheral populations of the perennial herb Gypsophila fastigiata. Oikos 99: 489-498.

Manton I (1937). The problem of Biscutella laevigata L. II. The evidence from meiosis. Ann Bot 51: 439-465.

Olowokudejo JD, Heywood VH (1984). Cytotaxonomy and breeding system on the genus Biscutella (cruciferae). Plant Syst Evol 145: 291-309.

Parisod C (2008). Postglacial recolonisation of plants in the western Alps of Switzerland. Bot Helv 118: 1-12.

Parisod C, Besnard G (2007). Glacial in situ survival in the Western Alps and polytopic autopolyploidy in Biscutella laevigata L. (Brassicaceae). Mol Ecol 16: 2755-2767.

Parisod C, Christin P-A (2008). Fine-scale association between genetic structure and environmental heterogeneity in a continuous population of Biscutella laevigata L. (Brassicaceae). New Phytol 178: 436-447.

Parisod C, Trippi C, Galland N (2005). Genetic variability and founder effect in the pitcher plant Sarracenia purpurea (Sarraceniaceae) in populations introduced into Switzerland: from inbreeding to invasion. Ann Bot 95: 277-286.

Petit RJ, Pineau E, Demesure B, Bacilieri R, Ducousso A, Kremer A (1997). Chloroplast DNA footprints of postglacial recolonization by oaks. Proc Nat Acad Sci USA 94: 9996-10001.

Petit RJ, Aguinagalde I, De Beaulieu JL, Bittkau C, Brewer S, Cheddadi $\mathrm{R}$ et al. (2003). Glacial refugia: hotspots but not melting pots of genetic diversity. Science 300: 1563-1565.

Raffl C, Schoenswetter P, Erschbamer B (2006). 'Sax-sess' genetics of primary succession in pioneer species on two parallel glacier forelands. Mol Ecol 15: 2433-2440.

Schönfelder P (1968). Chromozomenzahlen einiger Arten des Gattung Biscutella L. Öst Bot Zeit 115: 363-371.

Slatkin M (1993). Isolation by distance in equilibrium and nonequilibrium populations. Evolution 47: 264-279.

Thioulouse J, Chessel D, Doledec S, Olivier JM (1998). ADE-4. Université Lyon 1: Villeurbanne.

Thomas CD, Bodsworth EJ, Wilson RJ, Simmons AD, Davies ZG, Musche M et al. (2001). Ecological and evolutionary processes at expanding range margins. Nature 411: 577-581.

Vekemans X, Hardy OJ (2004). New insights from fine-scale spatial genetic structure analyses in plant populations. Mol Ecol 13: 921-935.

Yang S, Bishop JG, Webster MS (2008). Colonization genetics of an animal-dispersed plant (Vaccinium membranaceum) at Mount St Helens, Washington. Mol Ecol 17: 731-740.

Zimmermann NE, Kienast F (1999). Predictive mapping of alpine grasslands in Switzerland: species versus community approach. J Veget Sci 10: 469-482.

Supplementary Information accompanies the paper on Heredity website (http://www.nature.com/hdy) 